\title{
Meniscal allograft transplantation using a novel all-arthroscopic technique with specifically designed instrumentation
}

\author{
YA-DONG ZHANG, SHU-XUN HOU, HONG-BIN ZHONG, YI-CHAO ZHANG and DIAN-ZHONG LUO \\ Department of Orthopedics, The First Affiliated Hospital of PLA General Hospital (304 Hospital), Beijing 100048, P.R. China
}

Received March 31, 2016; Accepted September 19, 2017

DOI: $10.3892 /$ etm.2018.5766

\begin{abstract}
The present study describes a novel all-arthroscopic technique for medial and lateral meniscal allograft transplantation (MAT). Surgical instruments were specifically designed to assist in the all-arthroscopic approach for MAT. The bone plug attachment technique, either the arthroscopic-assisted or all-arthroscopic approach, attaches bone plugs to the anterior and posterior horns. In the present study, two sets of surgical implements were designed: One to produce bone plugs of predefined sizes in the anterior and posterior horns of the allograft meniscus (bone plug implements) and a second to create bone tunnels in the receptor tibial plateau to hold the bone plugs (bone tunnel implements). The present study demonstrated that an all-arthroscopic approach to MAT was feasible. Furthermore, the specifically designed surgical instruments allowed for consistent preparation of grafts and recipient tissues, contributing to a standardized approach to MAT. The present findings indicate that an all-arthroscopic approach to MAT may be achievable. They also provide the incentive for future clinical studies to directly compare the outcomes and to initiate the standardization of the procedure to optimize MAT and maximize patient outcomes and quality of life.
\end{abstract}

\section{Introduction}

The menisci are among the most commonly injured body parts, with $>1.7$ million patients undergoing meniscal surgery each year worldwide (1). In cases of meniscal injury, the aim is to preserve the meniscus whenever possible; however, many tears are either not repairable or the repair ultimately fails (2). A previous study reported a failure rate of $23.1 \%$ (3).

Correspondence to: Dr Ya-Dong Zhang or Dr Shu-Xun Hou, Department of Orthopedics, The First Affiliated Hospital of PLA General Hospital (304 Hospital), 51 Fucheng Road, Beijing 100048, P.R. China

E-mail: yadongzhang73@sina.com

E-mail: drhsx08@yeah.net

Key words: meniscal allograft transplantation, arthroscopy, all-arthroscopic, knee, instrument
In cases of untreatable or failed meniscal surgery, meniscectomy is required; however, this method of treatment is cautiously decided upon due to the certainty of progressive pain, loss of function and development of osteoarthritis (OA) caused by the altered biomechanical and biochemical environment $(2,4,5)$. Although still relatively rare compared with other types of orthopedic surgery, meniscal allograft transplant (MAT) is a powerful tool for orthopedic surgeons that is gaining popularity due to the known complications of meniscectomy (6).

Various MAT techniques have been developed since the first MAT was performed in 1984, including arthroscopic-assisted methods $(5,7,8)$. Controversies persist, however, regarding the optimal techniques for what is still viewed by some as a 'salvage procedure' $(5,9)$. Furthermore, MAT has been associated with short-term failure ( $\sim 4$ years) at a rate of $10.6 \%$ and a high complication rate of $13.9 \%$ (2). Consequently, additional efforts to improve the MAT technique and technology are warranted.

The purpose of the present study was to describe a novel, all-arthroscopic MAT technique and demonstrate the effect of associated instruments that were specifically designed for this procedure. It is anticipated that an all-arthroscopic method may assist in standardizing the procedure and ultimately shorten surgical times by making a technically demanding procedure easier, reduce intraoperative blood loss and improve short- and long-term outcomes.

\section{Materials and methods}

Ethical statement. The present study was approved by the Ethics Committee of the First Affiliated Hospital of PLA General Hospital (Beijing, China). Informed, written consent was obtained from all patients for the present study.

Instrumentation. Two sets of surgical implements were designed: One to produce bone plugs of predefined sizes in the anterior and posterior horns of the allograft meniscus (bone plug implements) and a second to create bone tunnels in the receptor tibial plateau to hold the bone plugs (bone tunnel implements).

The bone plug implements included a 1.8-mm guide pin and hollow drill with a conical inner diameter varying 5-8.5 mm, with the conical portion extending $15 \mathrm{~mm}$ in length. The drill had a hollow inner diameter of $2 \mathrm{~mm}$ to permit passage of the 
1.8-mm guide pin. The bone tunnel implements included an eccentric bone drill (with a $2.5-\mathrm{mm}$ bone cutting head on the end), obturators and attachment bolts.

Allograft preparation. Between March 2014 and March 2015, 24 patients (16 males and 8 females) with a mean age of 27.5 years (range, 17-48 years) participated in the present study. The inclusion criteria were meniscal deficiency defined by the presence of $<5 \mathrm{~mm}$ of uninterrupted circumferential hoop fibers as determined by preoperative MRI and confirmed by diagnostic arthroscopy. Exclusion criteria were inflammatory joint disease, unresolved or recent septic arthritis, metabolic or crystal disorders, body mass index $>35 \mathrm{~kg} / \mathrm{m}^{2}$, and deficient soft tissue coverage or tibiofemoral malalignment more than $2-3^{\circ}$. Residual synovial/soft tissue and any bone connected to the anterior and posterior horns of the allograft meniscus were removed. Disconnected bones on the anterior and posterior horns of the allograft meniscus required two precisely sized bone plugs with a diameter of $8.5 \mathrm{~mm}$ designed to match corresponding bone tunnels in the tibial plateau. The $1.8-\mathrm{mm}$ guide pin was positioned in the center of the footprint of the posterior horn of the meniscus, and the guide pin was maintained either at an outward angle of $15^{\circ}$ and a forward angle of $45^{\circ}$ (posterior horn), or backward angle of $30^{\circ}$ (anterior horn) relative to the plane of the tibial plateau (Fig. 1). The conical hollow drill was used, following the guide pin to drill from the side of the bone piece into the horn of the meniscus until the hollow drill reached the posterior horn of the meniscus, without damaging the horn of the meniscus (Fig. 2). Bone nibbling forceps were used to trim the residual bone from around the bone plug. The optimum bone plug was $13 \mathrm{~mm}$ in length, which closely matched the length of the bone groove in the tibial plateau $(15 \mathrm{~mm})$. Conical in shape, the diameter of the bone plug closest to the meniscus was $8.5 \mathrm{~mm}$ and the free end of the bone plug was $5 \mathrm{~mm}$ in diameter (Fig. 3). Once the guide pin was removed to produce the bone plug, a non-absorbable traction suture (TS1) was used to suture the attachment area on the posterior horn of the meniscus and the suture was passed through the hole made by the guide pin. A 2-0 TiCron nonabsorbable braided suture (Medtronic, Fridley, MN, USA) or an Ethibond suture (Ethicon, LLC, Somerville, NJ, USA) was used for TS3/TS4 and TS1/TS2, respectively. The meniscal tissue near the meniscal allograft bone plug was also securely sutured to fix the bone plug within the tibial tunnel (Fig. 4). A traction suture (TS2) was similarly passed through the bone plug, which was produced using a similar method on the anterior horn of the meniscus. The traction sutures aided the bone plug through the tunnel in the tibial plateau and through the exit on the inner side of the tibial tuberosity. Tension was maintained on TS1 and 2, which were tied together to attach the anterior and posterior horns of the meniscus. If the patient was young and energetic, screws were used to suspend and attach the traction suture to the anterior and posterior horns to increase stability.

A 2-0 nonabsorbable braided suture was used to create two additional traction sutures at the junction of both the anterior and posterior horns and the body of the meniscus (TS3 and 4). Once the meniscal allograft was implanted in the joint cavity, the sutures were passed through the joint capsule to assist with pulling the meniscal allograft into the

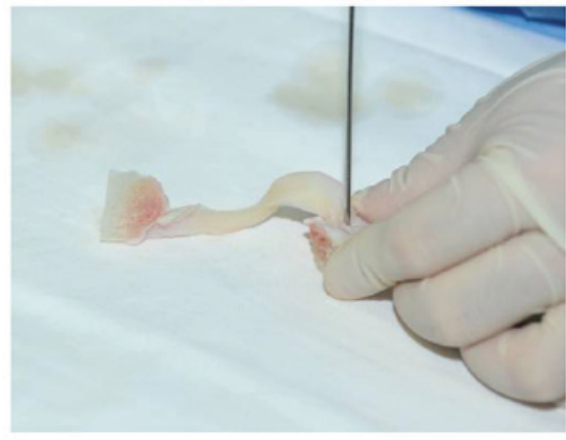

Figure 1. Positioning of the center of the footprint of the horns of the meniscus. Guide pin (1.8-mm) was positioned in the exact center of the footprint of the posterior horn of the meniscus and maintained at an outward angle of $15^{\circ}$ and a forward angle of $45^{\circ}$ (posterior horn) or a backward angle of $30^{\circ}$ (anterior horn) relative to the plane of the tibial plateau. The center of the bone piece on the posterior horn of the allograft meniscus was drilled.

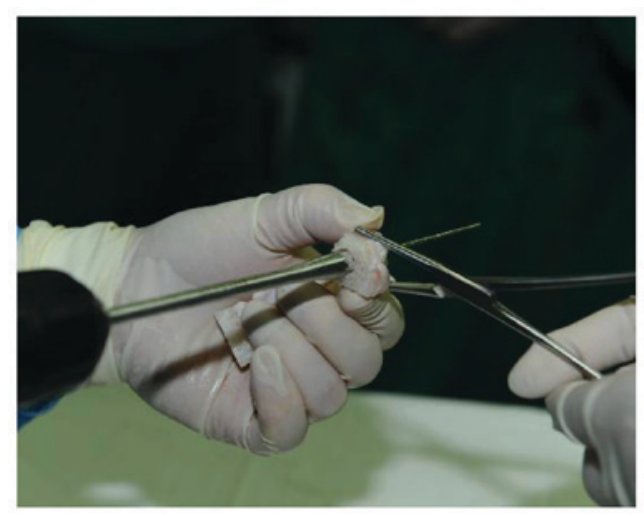

Figure 2. Two one plugs were produced with standard dimensions. Conical hollow drill (with an inner diameter of 5-8.5 mm and a 15-mm long conical portion) followed the guide pin to drill from the side of the bone piece into the horn of the meniscus. Once the posterior horn of the meniscus was reached, drilling was stopped. The horn of the meniscus was not damaged.

joint cavity and preventing the meniscus from springing back, ensuring the meniscus arrived at the correct position (Fig. 5). The sutures ultimately passed through the bone tunnels to the dorsal surface of the tibia and exited on the inside of the tibial tuberosity. TS3 and 4 had a certain amount of tension, as the surgeon could feel resistance coming from the meniscus and were tied to TS1 and 2.

Arthroscopic MAT. Once general anesthetic was induced in the patients in dorsal recumbency, a tourniquet was applied to the affected limb, and the knee was flexed at a $90^{\circ}$ angle off the side of the bed. Two incisions were made at the dorsomedial and dorsolateral aspects of the knee. Depending on the side of the meniscal transplant, either the medial or lateral incision was extended slightly to $1-1.5 \mathrm{~cm}$ in length. An auxiliary incision to protect the common peroneal nerve was created by the lateral meniscal transplant. Additionally, an incision was made to loosen the collateral ligament to expand the joint gap if it was too narrow.

Routine arthroscopic examination of the joint was performed and the pathology confirmed using standard techniques (10-13). If the tibial intercondylar eminence was 


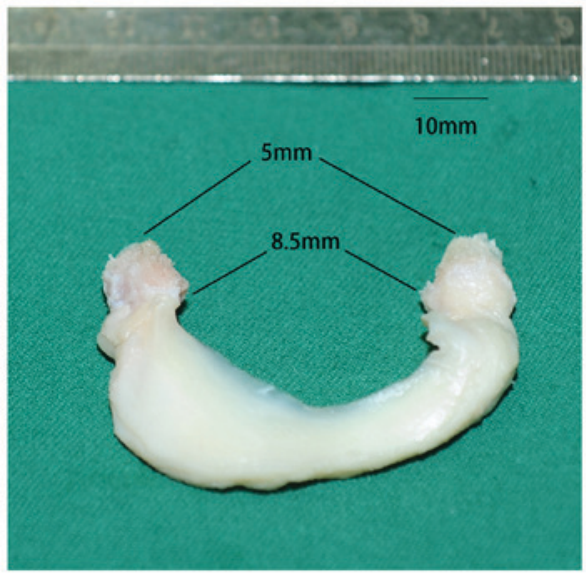

Figure 3. A conical bone plug. The diameter of the bone plug where it is closest to the meniscus was $8.5 \mathrm{~mm}$, and the free end of the bone plug had a diameter of $5 \mathrm{~mm}$.

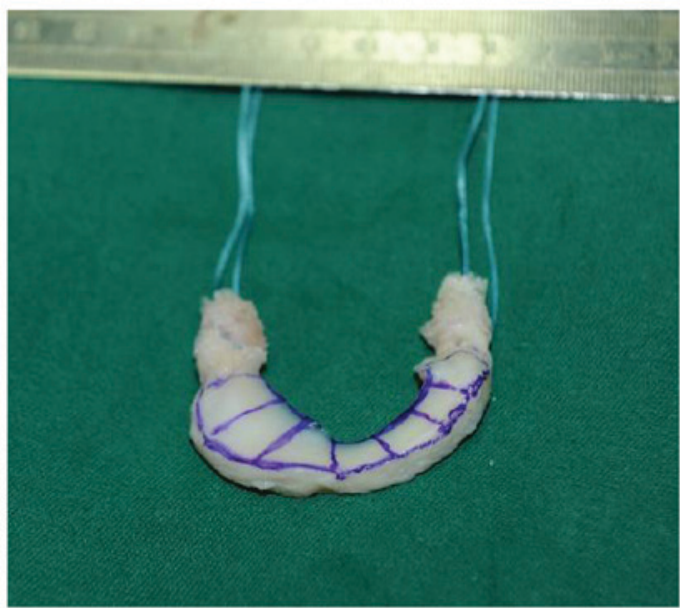

Figure 4. Nonabsorbable traction sutures were used on the posterior horn of the meniscus. Sutures were passed through the hole made by the guide pin. The meniscal tissue near the meniscal allograft bone plug was sutured, and Meilan marks were used to help determine the location of the upper and lower surfaces of the meniscus when it was in the joint cavity.

prominent, 4-6 $\mathrm{mm}$ of the affected side of the tibial intercondylar eminence and a small section $(\sim 4 \mathrm{~mm})$ of the central portion of the femoral condyle was removed prior to surgery to clearly expose the posterior horn of the receptor meniscus and facilitate surgical manipulation by performing intercondylar notchplasty. Soft tissue debridement was performed until the center of the posterior horn of the meniscus was clear, which in five cases necessitated the severing of the proximal aspect of the collateral ligament from the femoral condyle. Following surgery, screws were used to reattach the ligament. The deeper layers of the collateral ligament were not completely removed to avoid the capsule from 'ballooning out' and complicating the suturing of the meniscus.

The residual receptor meniscus was filed and planed until a rough, even margin was produced and bleeding occurred. Retention of a $2 \mathrm{~mm}$ residual meniscus margin aided in confirming that the anterior and posterior horns were transplanted to the correct locations, and suturing the transplanted meniscus to the margin of the stump aided in

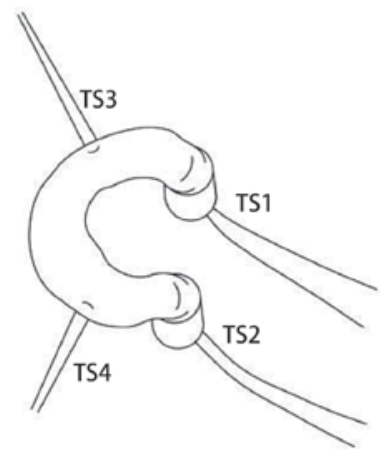

Figure 5. Traction sutures in the anterior and posterior horns of the meniscus were produced. 2-0 non-absorbable braided sutures were used to create one traction suture (traction sutures 3 and 4 ) at the junction of the posterior horn and the body of the meniscus (1/3 of the way from the rear of the meniscus) as well as at the junction of the anterior horn and body of the meniscus (1/3 of the way from the front of the meniscus).

preventing outward herniation and false crimping of the meniscus (Fig. 6) (14-16).

For junior surgeons, aluminum foil was used to produce a template with dimensions similar to those of the transplant. Placing this template on the tibial plateau within the joint confirmed the location of the bone tunnel and determined whether the transplant was appropriately situated on the tibial plateau without any notable overhang.

Production of bone tunnels in the tibial plateau. The anterior horn of the meniscus was attached to the forward slope of the tibia and the location of the anterior horn of the original meniscus indicated correct positioning. A guide pin was used to drill into the center of the anterior horn. A 9-mm hollow drill followed the guide pin to create a bone tunnel $\sim 15 \mathrm{~mm}$ deep. A triangular drill guide was placed in the bone tunnel and a guide pin was used to drill into the bottom of the blind bone tunnel from the cortical bone on the dorsomedial aspect of the tibia. The bone tunnel was expanded by $3 \mathrm{~mm}$ and a guide wire was passed through the cortical bone of the tibia and into the blind bone tunnel. The wire was grasped for subsequent use where it exited the knee joint.

The bone tunnel for the posterior horn was created by drilling a hole backwards from the tibial plateau. A 4-cm incision in the skin lateral to the patella near the tibial tuberosity was created and an anterior cruciate ligament (ACL) guide was inserted into the joint cavity. The entrance to the drilled hole was on the rear slope on the inside of the tibial tuberosity and in the center of the inner aspect of the posterior horn of the meniscus (Fig. 7A and B). Using the guide, a guide pin was drilled into the center of the footprint of the posterior horn of the receptor meniscus via the inside of the tibial tuberosity for the medial meniscus transplant or outside for the lateral meniscus transplant. A 4-mm hollow drill was used to expand the bone tunnel (Fig. 7C). The eccentric bone drill was inserted into the bone tunnel, and bone cutting head $(2.5-\mathrm{mm}$ in size) was passed through the bone tunnel and into the joint cavity. Subsequently, an obturator was inserted into the bone tunnel to ensure that the bone cutting head at the end of the eccentric bone drill was completely off-center relative to the bone tunnel and a bolt was used to attach the eccentric bone 


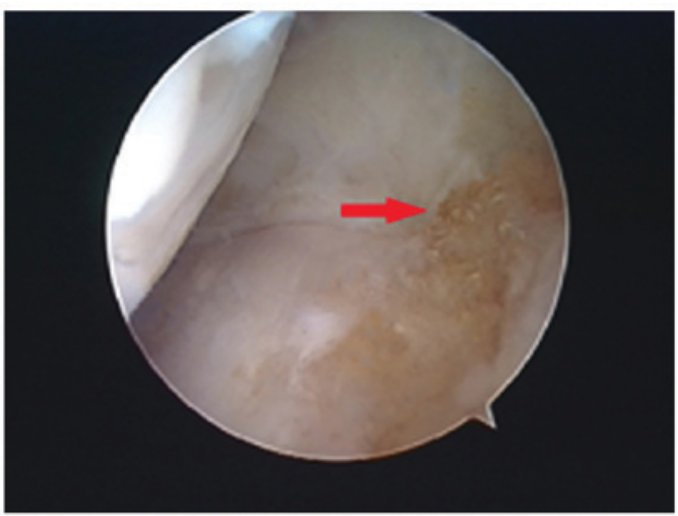

Figure 6. Residual receptor meniscus debridement was performed to the synovial margin. Approximately $2 \mathrm{~mm}$ of the residual meniscus was retained to help confirm that the anterior and posterior horns were transplanted to the correct locations (arrow).

drill and obturator, which formed an eccentric bone drill head. The eccentric bone drill head was turned using a power drill and removed while rotating in reverse $\sim 15 \mathrm{~mm}$ in the direction of the cortical bone at the front of the tibia, which formed a cavity that allowed for the accommodation of the bone plug (diameter, $9 \mathrm{~mm}$; the diameter was $(4+2.5 \times 2 \mathrm{~mm})$; length, $15 \mathrm{~mm}$ ) (Fig. 8A-F). Subsequently, the bolt was loosened, and the obturator and eccentric bone drill were removed in sequence. A guide wire was inserted into the bone tunnel and was pulled out from the anterior incision where guide wire of the anterior horn of the meniscus had been pulled out.

Inserting the meniscal allograft. TS1 and 2 were passed to the steel guide wire in the anterior and posterior horns. The steel guide wire was removed so that TS1 and 2 pulled the bone plugs of the anterior and posterior horns into the joint cavity through the skin incision to the bone tunnels in the tibial plateau to accommodate the anterior and posterior horns. The bone plugs on the anterior and posterior horns of the transplanted meniscus remained in the bone tunnels, and TS1 and 2 were pulled into the inner side of the tibial tuberosity on the dorsal surface of the tibia. A suture grasper (Acufex; Smith and Nephew plc, London, UK) pulled the suture at the junction of the horns and the body of the meniscus (TS3 and 4) out through the capsule and skin temporarily.

The knee joint was flexed at an angle of 20 and the knee was turned to facilitate passage of the bone plug on the posterior horn of the transplant. TS1 and 3 were pulled to guide the meniscus into the joint cavity, causing the posterior horn to enter the rear chamber of the joint cavity. In cases where it is difficult to pull TS1 and 3 to guide the posterior horn of the meniscus to enter the rear chamber of the joint cavity, a probe was used to push aside the posterior cruciate ligament and a hook was used to gently pull the posterior horn of the allograft meniscus to allow the bone plug to pass through. The posterior horn of the meniscus was prevented from entering the bone tunnel in the tibia and only the bone plug on the posterior horn was allowed to enter the bone tunnel to ensure the graft did not become too short. This procedure was repeated with TS2 and 4 in order to maneuver the entire graft into the joint, ensuring each horn entered the appropriate bone
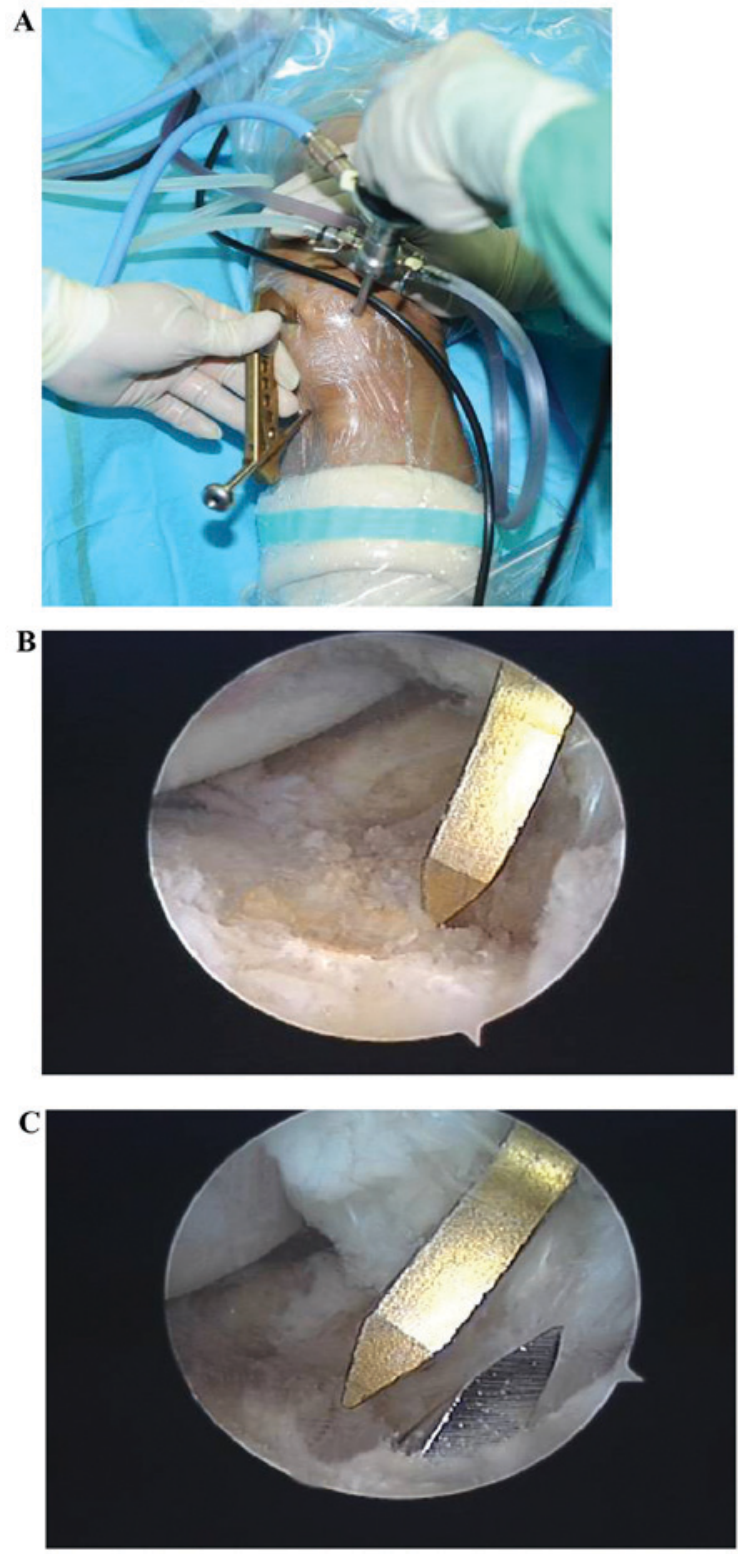

Figure 7. Production of the bone tunnel of the posterior horn of the receptor meniscus. (A and B) An anterior cruciate ligament guide was used to assist the drilling of the guide pin into the exact center of the posterior horn footprint of the receptor meniscus at the tibial tuberosity. (C) A 4-mm hollow drill was used to expand the bone tunnel.

tunnel. The meniscus was adjusted to its normal location under arthroscopic observation.

To confirm the appropriate position and location of the meniscus, the knee was straightened. Subsequently, the ends of TS1 and 2 were tied together, pulling the anterior and posterior horns of the meniscus outside of the cortical bone on the inside of the tibial tuberosity. This method ensured that the horns could be pulled tight and were able to enter the bone tunnels on the tibial plateau. The locations of TS3 and 4 at the junction of the horns and body of the meniscus were confirmed by locating the horn junctions and the body of the residual former meniscus. Two bone tunnels $\sim 2 \mathrm{~mm}$ in diameter were drilled on the dorsal surface of the tibia toward the two traction sutures at the edge of the joint cartilage on the tibial plateau. A steel guide wire was inserted and the traction sutures (TS3 and 4) were pulled through the bone tunnels in 

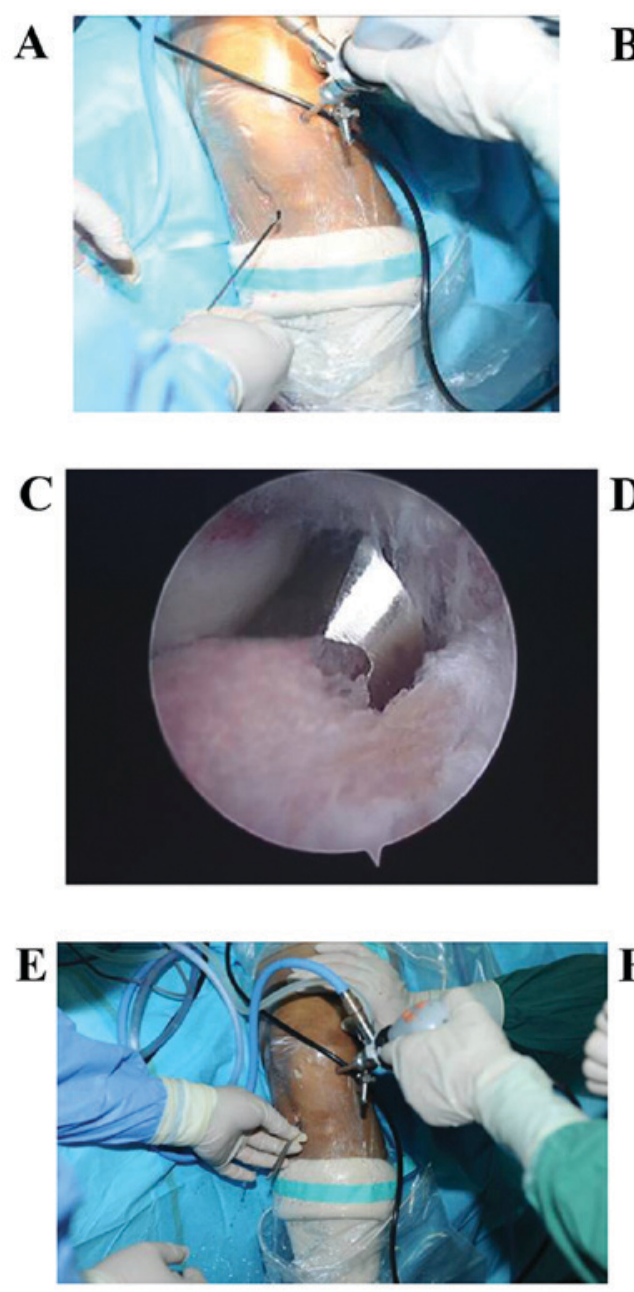

$\mathbf{B}$

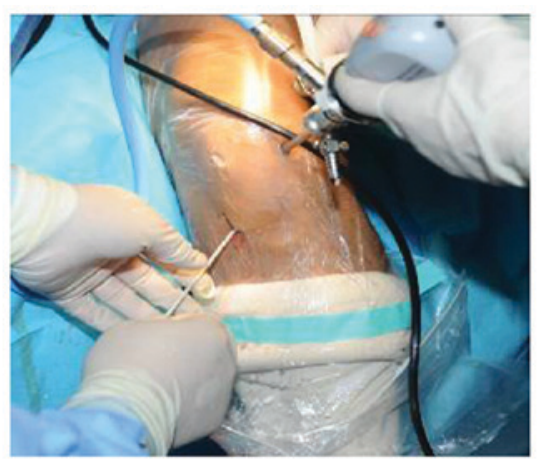

D

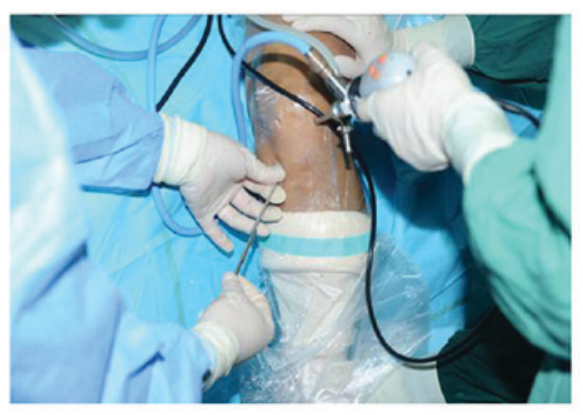

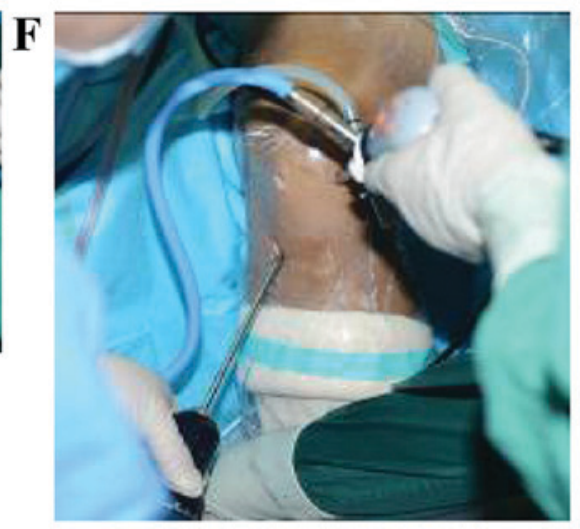

Figure 8. Accommodation of the posterior horn of the meniscal allograft was produced. (A) An eccentric bone drill was inserted into the bone tunnel; (B and C) the cutting head (eccentricity of $2.5 \mathrm{~mm}$ ) passed through the bone tunnel and into the joint cavity; (D and E) an obturator was inserted into the bone tunnel to ensure that the eccentric bone cutting head at the end of the eccentric bone drill was completely off-center relative to the bone tunnel. (A) Bolt was used to attach the eccentric bone drill and obturator to form an eccentric bone drill head. $(\mathrm{F})$ The eccentric bone drill head was turned using a power drill to pull out the eccentric bone drill $\sim 15 \mathrm{~mm}$ in the direction of the tibial cortical bone to form a cavity that accommodated a bone plug with a diameter of $9 \mathrm{~mm}$.

the edge of the joint cartilage on the tibial plateau to the outlet of the bone tunnel on the front surface of the tibia. Tension was maintained on the traction sutures (TS3 and 4) and these were tied to the traction sutures (TS1 and 2) holding the anterior and posterior horns of the meniscal allograft at the front surface of the tibia. This resulted in all four traction sutures anchoring the four attachment points of the allograft. The present approach allowed for TS3 and 4 to attach the synovial margin of the meniscal allograft, re-established some of the function of the coronary ligament between the meniscus and the edge of the tibial plateau and reduced the risk of herniation of the meniscal allograft.

Suturing the allograft. Standard meniscal suture repair methods were employed to suture the margin of the meniscus to the capsule and attach the meniscal allograft $(5,16)$. Vertical sutures are typically employed to attach the meniscus from the rear $1 / 3$ to the front in all cases and this was performed in the present study. A 2-0 non-absorbable suture (Ethibond) was used to attach the upper and lower surfaces of the meniscus with an alternating pattern. The meniscus was sutured directly to the meniscal bed (Fig. 9A and B) and it was confirmed by observation that the meniscal allograft was attached to the dissected portion of the tibia and the margin of the residual meniscus, which created tension around the circumference of the meniscus (Fig. 9C). A commonly used all-inside suturing method, including the use of the FasT-Fix Suture System (Smith and Nephew plc), was used to create two sutures on the posterior horn (with a low pulling resistance) and the remaining 6-8 sutures employed an inside-out vertical approach (17-20). Furthermore, a no. 18 spinal bone marrow puncture needle was used to suture the anterior horn with an outside-in pattern, using 0 braided nonabsorbable suture material, or open sutures (21-23). When necessary, bone anchors were used to attach the margin of the meniscus to the margin of the tibial plateau, which ensured the meniscus was 

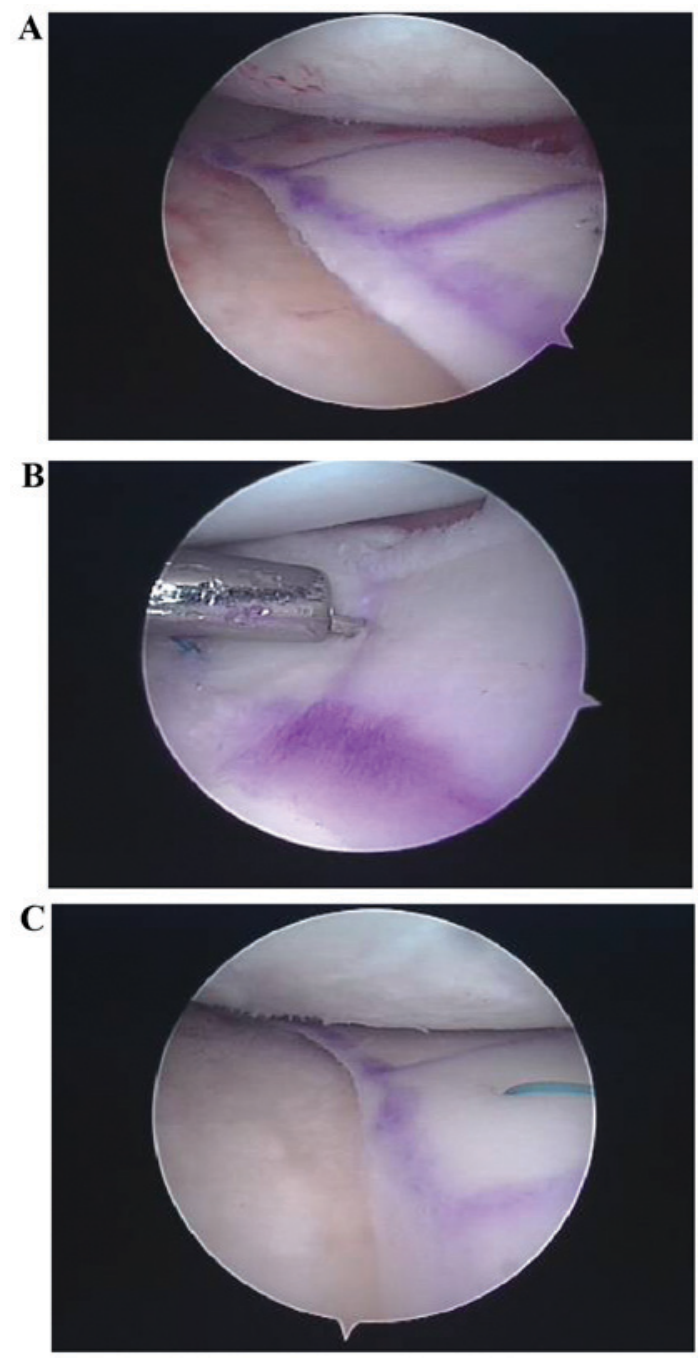

Figure 9. Suturing of the meniscus allograft. (A) Once the anterior and posterior horns were attached. (B) Vertical sutures were attached to the meniscus from the rear $1 / 3$ to the front. Upper and lower surfaces of the meniscus were attached with 2-0 nonabsorbable sutures with an alternating pattern. (C) The meniscus was directly sutured to the meniscus bed.

securely attached. Prior to suturing the meniscus, incisions in the medio- or lateroplantar aspect of the joint were created, which facilitated an inside-to-outside pattern. Notably, when performing a lateral meniscal transplant, a 3-cm long incision at the lateroplantar aspect of the joint should be created to avoid injury to the common peroneal nerve (located plantar to the inner collateral ligament and the dorsal to the sartorius). In the present study, the pesanserinus tendon was pulled in a plantar direction, blunt dissection of the semimembranosus tendon and the knee capsule was performed, and blunt separation of the gastrocnemius tendon and capsule was established, avoiding the infrapatellar branch of the saphenous nerve. Small curved forceps were used to separate the subcutaneous tissue and deeper tissue until the capsule was reached. When suturing the rear of the meniscus, the suture needle was pulled through the $3 \mathrm{~cm}$ incision. A meniscal spoon retractor was applied to prevent the inside-to-outside sutures from injuring nerves or blood vessels and the suture cord was tied directly above the capsule. Two or three small incisions were required to extract all of the meniscal sutures. Subsequently, the joint was examined while gently flexing and rotating prior to routine closure of the incisions and bandaging the limb.

\section{Results and Discussion}

A recent systematic review reported that the MAT procedure has matured from an open approach with femoral condyle osteotomy or distraction devices to the widely used arthroscopically-assisted procedure with miniarthrotomy (4). Given the benefits of minimally invasive surgery and desire to further develop MAT, the purpose of the present study was to describe a novel all-arthroscopic technique for performing meniscal MAT, either lateral or medial. In the present study, the 1.8-mm guide pin was positioned in the center of the footprint of the posterior horn of the meniscus and the eccentric bone drill was inserted into the bone tunnel. The eccentric bone drill head was turned using a power drill and removed while rotating in reverse $\sim 15 \mathrm{~mm}$ in the direction of the cortical bone at the front of the tibia, which formed a cavity that allowed for the accommodation of the bone plug (diameter, $9 \mathrm{~mm}$; the diameter was $(4+2.5 \times 2 \mathrm{~mm})$; length, $15 \mathrm{~mm})$. To the best of our knowledge, this is the first study to use this method. Although technically demanding, there are some benefits of minimally invasive orthopedic procedures, as they have a finer bone tibial tunnel compared with other methods (24). MAT is a procedure that has gained interest since its inception in the early 1980s (25). However, despite being performed for over three decades, controversy persists regarding several of the finer aspects of MAT, including the following: Indications for surgery and patient selection, including age, symptoms and timing of surgery; graft preservation technique; fixation technique (bony fixation vs. an all-suture technique); postsurgical rehabilitation procedures; appropriate outcome measures, including Lysholm score, International Knee Documentation Committee (IKDC) subjective knee form, Tegner activity level score, Fulkerson questionnaire, WOMAC index, and the Kellgren and Lawrence OA grade; the impact of concomitant surgical procedures; impact on OA; and what is considered a successful surgery. These controversies continue to be salient in this field considering the growing number of MAT procedures performed annually together with the lack of controlled clinical studies and long-term follow-up data $(2,4,6)$.

The all-arthroscopic MAT technique described in the present study demonstrated a primary step to pioneer a standardized procedure using specifically designed instruments for creating bone plugs and tunnels. The present approach, rather than the bone bridge approach, was selected as the literature has revealed that the bone insertion technique offers a biodynamic advantage (26-30). Previous studies have also indicated that the reliable attachment of the anterior and posterior horns of the meniscus to precisely dissected locations on the receptor is a key surgical determinant of postoperative meniscal function $(28,31,32)$. If the anterior and posterior horns are not securely attached, poor meniscal function may cause widespread deterioration of the cartilage in load-bearing areas of the joint $(27,33)$. Overall, it is currently accepted that bone attachment of the anterior and posterior horns of the meniscus is the gold standard for MAT $(34,35)$.

The bone plug attachment technique, either the arthroscopic-assisted or all-arthroscopic approach, attaches 
one bone plug to the anterior and posterior horns, respectively. Several advantages are associated with this method, including little trauma to the tibial plateau, little requirement to remove bone, the preservation of the tibial intercondylar eminence and adjustment of the meniscus location depending on allograft size and maintenance of optimal meniscal allograft tension $(8,36)$. However, the technique is difficult and time consuming and the distances and locations of the anterior and posterior horns may be inaccurate (37). Meniscus transplant abnormalities may occur due to error in the location of tibial tunnels (38). For example, a large bone tunnel diameter may increase the risk of injury to the receptor tibial plateau and increase the risk of tibial plateau fracture. This is particularly prominent for patients requiring concomitant surgical procedures to the ACL (39).

Presently, one of the largest concerns with MAT is the lack of standard surgical implements, which has forced surgeons to depend on their hands and eyes alone to produce the bone plugs and bone tunnels. As a result, bone plugs and tunnels are frequently inconsistent in size and/or shape, requiring repeated corrections that prolong surgery time and reduce surgical accuracy, potentially achieving suboptimal attachment of the anterior and posterior horns of the meniscus.

The development of the surgical implements described in the present study was anticipated to facilitate bone plug creation, implantation and attachment, minimize trauma to the tibial plateau caused by the bone plug attachment technique and reduce the risk of postsurgical complications, including tibial fracture. In addition, such instrumentation may promote surgical standardization, minimize trauma, simplify bone tunnel production, enhance bone plug attachment quality, shorten surgical times and decrease intraoperative bleeding among other potential benefits $(37,40)$.

The success of the bone plug attachment technique depends on correct dissection and maintenance of annular tension. Previous studies have indicated that crimping due to protrusion of the margin of the meniscus, or even subluxation, may occur following meniscal transplantation (15). The meniscus is typically attached to the tibial plateau via the tibia ligaments, not by the capsule. Consequently, it is crucial for the meniscus to be attached to the dissected portion of the tibia and to the margin of the residual meniscus. In the procedure described in the present study, a steel guide wire was used to pull the traction sutures to the junction between the posterior horn and the body of the meniscus, and the junction between the anterior horn and the body of the meniscus to the inside of the tibial tuberosity, while maintaining appropriate tension when tying the sutures outside the cortical bone (inside the tibial tuberosity). The sutures attached to the anterior and posterior horns formed four attachment points, simplifying meniscal implantation and preventing the meniscus from protruding. Furthermore, it is hypothesized that the attachment of a meniscal allograft to the synovial margin may allow a certain degree of reconstruction of the function of the coronary ligament joining the meniscus and margin of the tibial plateau because the sutures (TS3 and 4) were applied to fix the meniscus to the margin of the tibial plateau, which may stabilize the margin of the meniscal allograft, increase the stability of the meniscus and largely avoid risk of meniscal herniation and/or dislocation (24). This approach is also supported by Stone and Walgenbach (24), who noted that out of three attachment methods, a four-point attachment method that involved the anterior and posterior horns and the junctions employed between the posterior horn and the body meniscus achieved the greatest meniscal stability, which prevented bulging and improved the accuracy of meniscal positioning. Thus, the surgery was successfully performed without any complications of meniscal herniation and/or dislocation in all cases.

The present study comprehensively described a novel bone all-arthroscopic technique and instrumentation for MAT. The present findings provided preliminary results for future clinical studies, which are required to identify the outcomes associated with the present technique. Those outcomes may then be compared with other studies to assist in improving MAT. Furthermore, although the present indicates the use of the surgical implements in an inner meniscal transplantation, the described technique may also be adapted for use in outer meniscal transplantations $(8,37)$.

\section{References}

1. Samitier G, Alentorn-Geli E, Taylor DC, Rill B, Lock T, Moutzouros V and Kolowich P: Meniscal allograft transplantation. Part 1: Systematic review of graft biology, graft shrinkage, graft extrusion, graft sizing, and graft fixation. Knee Surg Sports Traumatol Arthrosc 23: 310-322, 2015.

2. Smith NA, MacKay N, Costa M and Spalding T: Meniscal allograft transplantation in a symptomatic meniscal deficient knee: A systematic review. Knee Surg Sports Traumatol Arthrosc 23: 270-279, 2015.

3. Nepple JJ, Dunn WR and Wright RW: Meniscal repair outcomes at greater than five years: A systematic literature review and meta-analysis. J Bone Joint Surg Am 94: 2222-2227, 2012.

4. Myers $P$ and Tudor F: Meniscal allograft transplantation: How should we be doing it? A systematic review. Arthroscopy 31: 911-925, 2015.

5. Smith NA, Costa ML and Spalding T: Meniscal allograft transplantation: Rationale for treatment. Bone Joint J 97-B: 590-594, 2015.

6. Samitier G, Alentorn-Geli E, Taylor DC, Rill B, Lock T, Moutzouros V and Kolowich P: Meniscal allograft transplantation. Part 2: Systematic review of transplant timing, outcomes, return to competition, associated procedures, and prevention of osteoarthritis. Knee Surg Sports Traumatol Arthrosc 23: 323-333, 2015.

7. Jang KM and Wang JH: Lateral meniscus allograft transplantation using a single-incision technique. Knee Surg Sports Traumatol Arthrosc 23: 258-263, 2015.

8. Lubowitz JH, Verdonk PC, Reid JB and Verdonk R: Meniscus allograft transplantation: A current concepts review. Knee Surg Sports Traumatol Arthrosc 15: 476-492, 2007.

9. Lubowitz JH: Editorial commentary: Meniscal allograft yields acceptable outcomes (for a salvage procedure). Arthroscopy 31: 926, 2015

10. Javidan P, Ahmed M and Kaar S: Arthroscopic release of the deep medial collateral ligament to assist in exposure of the medial tibiofemoral compartment. Arthrosc Tech 3: e699-e701, 2014.

11. Harato K, Niki Y, Nagashima M, Masumoto K, Otani T, Toyama Y and Suda Y: Arthroscopic visualization of abnormal movement of discoid lateral meniscus with snapping phenomenon. Arthrosc Tech 4: e235-e238, 2015.

12. Gauffiny $\mathrm{H}$, Tagesson S, Meunier A, Magnusson $\mathrm{H}$ and Kvist J: Knee arthroscopic surgery is beneficial to middle-aged patients with meniscal symptoms: A prospective, randomised, single-blinded study. Osteoarthritis Cartilage 22: 1808-1816, 2014.

13. Sihvonen R, Englund M, Turkiewicz A and Järvinen TL: Mechanical symptoms as an indication for knee arthroscopy in patients with degenerative meniscus tear: A prospective cohort study. Osteoarthritis Cartilage 24: 1367-1375, 2016.

14. Iñigo-Pavlovich R: Radiofrequency and meniscus. From excision to repair. Sports Med Arthrosc Rev 13: 193-197, 2005. 
15. Khetia EA and McKeon BP: Meniscal allografts: Biomechanics and techniques. Sports Med Arthrosc 15: 114-120, 2007.

16. Yoldas EA, Sekiya JK, Irrgang JJ, Fu FH and Harner CD: Arthroscopically assisted meniscal allograft transplantation with and without combined anterior cruciate ligament reconstruction. Knee Surg Sports Traumatol Arthrosc 11: 173-182, 2003.

17. Grant JA, Wilde J, Miller BS and Bedi A: Comparison of inside-out and all-inside techniques for the repair of isolated meniscal tears: A systematic review. Am J Sports Med 40 459-468, 2012

18. Turman KA, Diduch DR and Miller MD: All-inside meniscal repair. Sports Health 1: 438-444, 2009.

19. Nelson CG and Bonner KF: Inside-out meniscus repair. Arthrosc Tech 2: e453-e460, 2013.

20. Choi NH, Kim TH and Victoroff BN: Comparison of arthroscopic medial meniscal suture repair techniques: Inside-out versus all-inside repair. Am J Sports Med 37: 2144-2150, 2009.

21. Rodeo SA: Arthroscopic meniscal repair with use of the outside-in technique. Instr Course Lect 49: 195-206, 2000.

22. Kelly JD IV and Ebrahimpour P: Chondral injury and synovitis after arthroscopic meniscal repair using an outside-in mulberry knot suture technique. Arthroscopy 20: e49-e52, 2004.

23. Abdelkafy A, Aigner N, Zada M, Elghoul Y, Abdelsadek H and Landsiedl F: Two to nineteen years follow-up of arthroscopic meniscal repair using the outside-in technique: A retrospective study. Arch Orthop Trauma Surg 127: 245-252, 2007.

24. Stone KR and Walgenbach AW: Meniscal allografting: The three-tunnel technique. Arthroscopy 19: 426-430, 2003.

25. Milachowski KA, Weismeier K and Wirth CJ: Homologous meniscus transplantation. Experimental and clinical results. Int Orthop 13: 1-11, 1989.

26. Abat F, Gelber PE, Erquicia JI, Pelfort X, Gonzalez-Lucena G and Monllau JC: Suture-only fixation technique leads to a higher degree of extrusion than bony fixation in meniscal allograft transplantation. Am J Sports Med 40: 1591-1596, 2012.

27. Alhalki MM, Howell SM and Hull ML: How three methods for fixing a medial meniscal autograft affect tibial contact mechanics. Am J Sports Med 27: 320-328, 1999.

28. Chen MI, Branch TP and Hutton WC: Is it important to secure the horns during lateral meniscal transplantation? A cadaveric study. Arthroscopy 12: 174-181, 1996.

29. Paletta GA Jr, Manning T, Snell E, Parker R and Bergfeld J: The effect of allograft meniscal replacement on intraarticular contact area and pressures in the human knee. A biomechanical study. Am J Sports Med 25: 692-698, 1997.
30. Wang H, Gee AO, Hutchinson ID, Stoner K, Warren RF, Chen TO and Maher SA: Bone plug versus suture-only fixation of meniscal grafts: Effect on joint contact mechanics during simulated gait. Am J Sports Med 42: 1682-1689, 2014.

31. Bryan TK, Robert HB and Scott AR: Meniscal allograft transplantation: Surgical technique. Techniques in Knee Surgery 3: 8-18, 2004

32. Paletta GA Jr, Manning T, Snell E, Parker R and Bergfeld J: The effect of allograft meniscal replacement on intraarticular contact area and pressures in the human knee. A biomechanical study. Am J Sports Med 25: 692-698, 1997.

33. Lazovic D, Wirth CJ, Knösel T, Gossé F and Maschek HG: Meniscus replacement using incongruent transplants-an experimental study. Z Orthop Ihre Grenzgeb 135: 131-137, 1997 (In German)

34. Shelton WR and Dukes AD: Meniscus replacement with bone anchors: A surgical technique. Arthroscopy 10: 324-327, 1994.

35. Vaquero $\mathrm{J}$ and Forriol F: Meniscus tear surgery and meniscus replacement. Muscles Ligaments Tendons J 6: 71-89, 2016.

36. Farr J and Gersoff W: Current meniscal allograft transplantation. Sports Med Arthrosc Rev 12: 69-82, 2004

37. Farr J, Rawal A and Marberry KM: Concomitant meniscal allograft transplantation and autologous chondrocyte implantation: Minimum 2-year follow-up. Am J Sports Med 35: 1459-1466, 2007.

38. Sekaran SV, Hull ML and Howell SM: Nonanatomic location of the posterior horn of a medial meniscal autograft implanted in a cadaveric knee adversely affects the pressure distribution on the tibial plateau. Am J Sports Med 30: 74-82, 2002.

39. Zhang YD, Hou SX, Zhang YC, Luo DZ, Zhong HB and Zhang H: Arthroscopic combined medial and lateral meniscus transplantation after double-tunnel, double-bundle anterior cruciate ligament reconstruction in the same knee. Knee 19: 953-958, 2012

40. Faivre B, Boisrenoult P, Lonjon G, Pujol N and Beaufils P. Lateral meniscus allograft transplantation: Clinical and anatomic outcomes after arthroscopic implantation with tibial tunnels versus open implantation without tunnels. Orthop Traumatol Surg Res 100: 297-302, 2014. 